\title{
Hepatitis $C$ virus promotes hepatocellular carcinogenesis by targeting TIPE2, a new regulator of DNA damage response
}

\author{
Yaohui Wang ${ }^{1}$ - Yinan Jiang ${ }^{2}$. Jinxue Zhou ${ }^{3}$ - Wuhui Song ${ }^{4}$. \\ Jing $\mathrm{Li}^{1} \cdot$ Mingli Wang ${ }^{1} \cdot$ Jiuge Chen $^{1} \cdot$ Rui Xu ${ }^{1} \cdot$ Jingjing Zhang ${ }^{1}$. \\ Fanni Ma ${ }^{1}$ - Youhai H. Chen ${ }^{5}$. Yuanfang Ma ${ }^{1}$
}

Received: 13 April 2016/Accepted: 13 September 2016/Published online: 30 September 2016

(C) The Author(s) 2016. This article is published with open access at Springerlink.com

\begin{abstract}
Infection of hepatitis C virus (HCV) is associated with primary hepatocellular carcinoma (HCC). However, its underlying molecular mechanisms remain enigmatic. Tumor necrosis factor- $\alpha$-induced protein 8 -like 2 (TIPE2), a new negative regulator of immunity, plays significant roles in modulating inflammation and tumorigenesis. We hypothesized that TIPE2 might be involved in the development of HCVinduced HCC. To test this hypothesis, the expression of TIPE2 was determined by Western blot in the tumor and pericarcinomatous tissues collected from ten HCV-positive HCC patients; the interaction between TIPE2 and HCVencoded non-structural proteins was analyzed by immunoprecipitation and immunofluorescence assays, and tumorigenesis and its mechanisms were studied in cell models and nude mice. Our results demonstrated that the expression of TIPE2 was significantly reduced in HCC tissues compared to that in the paracarcinoma tissues. HCV-encoded non-structural
\end{abstract}

Yaohui Wang, Yinan Jiang, and Jinxue Zhou contributed equally

Yuanfang Ma

mayf@henu.edu.cn

1 Henan Key Laboratory of Engineering Antibody Medicine, Medical College of Henan University, Kaifeng, Henan 475004, China

2 College of Basic Medical Sciences, Zhengzhou University, Zhengzhou, Henan 450001, China

3 Zhengzhou University Affiliated Tumor Hospital, Zhengzhou, Henan 450001, China

4 Key Laboratory of Medical Molecular Virology, School of Basic Medical Sciences, Shanghai Medical College, Fudan University, Shanghai, China

5 Department of Pathology and Laboratory Medicine, University of Pennsylvania Perelman School of Medicine, Philadelphia, PA 19104, USA protein NS5A could specifically interact with TIPE2 and induce its degradation. Downregulation of TIPE2 by shRNA in cell lines increased genomic DNA damage and promoted cell colony formation in vitro and tumorigenesis in nude mice. In contrast, overexpression of TIPE2 had an opposite effect. Downregulation of TIPE2 by NS5A is associated with genomic DNA instability and HCV-induced HCC development. Thus, TIPE2 may be a new therapeutic target for the treatment of HCV-associated HCC.

Keywords Hepatitis C virus · Hepatocellular carcinoma . NS5A · TIPE2 - DNA damage response

$\begin{array}{ll}\text { Abbreviations } \\ \text { HCC } & \text { Hepatocellular carcinoma } \\ \text { HCV } & \text { Hepatitis C virus } \\ \text { DDR } & \text { DNA damage response } \\ \text { TIPE2 } & \text { Tumor necrosis factor- } \alpha \text {-induced protein- } 8 \text { like-2 } \\ \text { ROS } & \text { Reactive oxidative stress } \\ \text { NOS } & \text { Nitric oxide synthase }\end{array}$

\section{Introduction}

Currently, there are more than 150 million hepatitis $\mathrm{C}$ virus (HCV)-infected individuals in the world [1]. Chronic HCV infection is highly associated with hepatocellular carcinoma (HCC). HCC is the most common histological subtype of primary liver carcinoma and accounts for $70-85 \%$ of total liver cancers [1,2], and almost half of the patients are from China [3].

$\mathrm{HCV}$, a sense-strand RNA virus, replicates exclusively in the cytoplasm and is unable to integrate into the host genome; the underlying mechanisms of $\mathrm{HCV}$-induced hepatocarcinogenesis 
remain elusive. Among the ten $\mathrm{HCV}$ encoded structural (core, E1, E2) and non-structural (P7, NS2, NS3, NS4A, NS4B, NS5A, NS5B) proteins [4], core, NS3, NS4B, and NS5A have been shown to directly activate oncogenic molecular pathways and promote tumor formation in vivo [5-8]. The strategies employed by $\mathrm{HCV}$ and encoded proteins to induce tumor formation include chronic inflammation, reactive oxidative stress (ROS), steatosis, fibrosis, and so on. DNA damage/repair is associated with almost all of the above pathogenic patterns. In fact, core, NS3/4A, NS5A, and NS5B have been reported to enhance DNA damage or suppress damage repair [9-13]. Consistently, in HCC patients, accumulation of DNA damage has been detected in the peripheral blood lymphocytes [14] and abundant $\mathrm{H} 2 \mathrm{AX}^{+} \mathrm{T}$ lymphocytes were found in the liver [15].

Tumor necrosis factor- $\alpha$-induced protein- 8 like-2 (TIPE2 or TNFAIP8L2) is a newly identified protein essential for the maintenance of immune homeostasis [16]. The crystal structure of TIPE2 revealed a large hydrophobic central cavity as the binding sites for cofactors [17]. Apart from maintenance of immune homeostasis, TIPE2 inhibits Ras activity via binding RalGDS and thereafter suppresses Ras-induced tumorigenesis in mice [18]. Downregulation of TIPE2 is associated with poor prognosis of non-small cell lung cancer, and it can also inhibit HCC cell metastasis [19, 20]. A recent study showed that expression of TIPE2 was reduced in peripheral blood mononuclear cells and tumor tissues from HBV-infected patients compared to healthy individuals [21]. Interestingly, TIPE2 was also shown to negatively regulate oxidative burst, indicating a possible involvement of DNA damage in the TIPE2-mediated tumorigenesis [22]. However, whether TIPE2 and TIPE2-mediated DNA damage are involved in $\mathrm{HCV}$-related HCC is still unknown.

In the present study, we investigated the association between TIPE2 and HCV-related HCC at clinical specimen, cell culture, and animal model aspects. The results showed that expression of TIPE2 is significantly reduced in tumor tissues compared to that in the paracarcinoma tissues from HCVpositive HCC patients. HCV/NS5A interacts with TIPE2 and promotes its degradation. Ectopic expression of TIPE2 can reduce DNA damages, while silencing TIPE2 with small hairpin (shRNA) can enhance it. Upregulation of TIPE2 can inhibit the HCC's tumor characteristics. These results suggest that TIPE2 is a negative regulator of HCV-associated HCC.

\section{Materials and methods}

\section{Clinical specimens}

This study was approved by the Ethics Committees of the Medical College of Henan University, and written informed consent was obtained from all participants. Tumor and pericarcinomatous liver tissues were collected from ten
HCV-positive HCC patients at the Affiliated Tumor Hospital of Zhengzhou University. The tissues were frozen at $-80{ }^{\circ} \mathrm{C}$, and protein was extracted by radioimmunoprecipitation (RIPA) tissue lysis buffer after grinding in liquid nitrogen. Patients' clinical characteristics are presented in Table 1.

\section{Animals and cells}

The animal experiments performed in this study were prior approval by the Animal Experimentation Committee of Henan University. Four-week-old male BALB/cA-nu mice were purchased from Beijing HFK Bioscience. All mice received standard diet and water and were treated in accordance with the National Guide for the Care and Use of Laboratory Animals. The cell lines HEK293T, Changliver, and Huh7 cells were maintained in DMEM medium supplemented with $10 \%$ fetal bovine serum, $2 \mathrm{mM}$ L-glutamine, and penicillin (100 units/ml)-streptomycin $(100 \mu \mathrm{g} / \mathrm{ml})$.

\section{Antibodies and reagents}

Mouse anti-Chk1, rabbit anti-Chk2, anti- $\mathrm{H}_{2} \mathrm{AX}$, antiphospho-Chk1, anti-phospho-Chk2, and anti $\gamma-\mathrm{H}_{2} \mathrm{AX}$ antibodies were purchased from Cell Signaling Technology (Danvers, USA). Mouse monoclonal anti- $\beta$-actin and antiFLAG were purchased from Sigma (St. Louis, USA), and rabbit anti-TIPE2 was purchased from Boster (Wuhan, China). Puromycin, crystal violet, MTT, DMSO, and bovine serum albumin (BSA) were purchased from Sigma, and DMEM medium and fetal bovine serums were purchased from Gibco (Gran Island, USA). Lentiviral vector pLKO.1, psPAX2, and pMD2.G were obtained from Addgene.

Transfection and establishment of stable cell lines For transient transfection, the cells were inoculated overnight and reached to $60-70 \%$ confluence; a certain amount of plasmid DNA was transiently transfected into the cells with the XtremeGENE HP DNA transfection reagent following the manufacturer's protocol (Promega, Madison, USA). The cells were harvested $48 \mathrm{~h}$ post-transfection; they were subjected
Table 1 Patients' this study clinical characteristics in

\begin{tabular}{ll}
\hline & Patients \\
\hline Number & 10 \\
Mean age (years) & 65.6 \\
Gender(male/female) & $6 / 4$ \\
HCV (+) & $10 / 10$ \\
HBV (+) & $1 / 10$ \\
Mean AFP (ng/ml) & 725.13 \\
\hline
\end{tabular}

AFP Alpha-fetoprotein, $H B V$ hepatitis B virus 
to the indicated experiments. To generate stable cell lines, the Changliver cells were transduced with lentiviral particles containing either TIPE2 shRNA or scramble shRNA. For ectopic expression of TIPE2, the Huh7 cells were infected with lentiviral particles expressing TIPE2 cDNA or empty vector. The cells were selected in $2 \mu \mathrm{g} / \mathrm{ml}$ puromycin for 2 weeks and were pooled for future study.

\section{Cell proliferation and plate colony formation assay}

For plate colony formation assay, cells were detached and seeded in six-well plates with 500 cells per well in culture medium. Two weeks later, visible clone cell clusters appeared. The cells were washed twice with PBS and fixed in $4 \%$ paraformaldehyde for $15 \mathrm{~min}$ followed by staining with crystal violet for $20 \mathrm{~min}$. The cells were then washed with PBS and air-dried. The colony is quantified and photographed under microscopy. The colony number divided by total cell numbers accounts for the colony formation rate. For cell viability, the cells were seeded in 96-well plates at a density of $5 \times 10^{3}$ cells per well, and cell growth curve was measured by MTT assay.

\section{DNA damage model in vitro}

To construct DNA damage cell model, the cells were seeded into 12-well plates; $24 \mathrm{~h}$ later, culture medium was changed with fresh medium containing hydroxyurea or DMSO at $3 \mathrm{mM}$ and incubated for another $24 \mathrm{~h}$. Cell lysates were then collected for immune blotting analysis.

\section{Immunoblotting analysis and co-immunoprecipitation-}

Cells were lysed with RIPA buffer containing protease inhibitor cocktail, and protein concentration was determined using bicinchoninic acid reagent (CWBIO, Beijing, China). Equal amounts of protein were loaded in each well for electrophoresis followed by transferring onto nitrocellulose filter membrane. After blocking with $5 \%$ fat-free milk or $5 \%$ BSA, the membranes were incubated with primary antibodies overnight at $4{ }^{\circ} \mathrm{C}$. After washing in Tris-buffered saline and Tween (TBST) buffer for three times, the membranes were incubated with HRP-conjugated secondary antibody for $1 \mathrm{~h}$ at room temperature. Then, the membranes were washed in TBST for three to four times, and then, they were developed in ECL buffer for $2 \mathrm{~min}$. The signals were measured by exposing to the X-ray film. For co-immunoprecipitation, the cells were lysed in immunoprecipitation (IP) lysis buffer containing protease and phosphatase inhibitors. About $1 \mathrm{mg}$ proteins were incubated with proper primary antibody at $4{ }^{\circ} \mathrm{C}$ overnight (control $\mathrm{IgG}$ is used as negative control). Fifty microliters of $50 \%$ slurry protein A-agarose beads was added to the cell lysates for $2 \mathrm{~h}$. The protein A-agarose beads were pelleted and washed in TBST buffer for five times. The proteins that are eluted from protein A-agarose beads were subjected to the immunoblotting. For glutathione S-transferase (GST) pulldown assay, the cells were lysed in the IP lysis buffer. The equal amounts of lysated proteins were incubated with GST alone or GST-TIPE2 proteins that were purified from bacteria at $4{ }^{\circ} \mathrm{C}$ overnight. Fifty microliters of glutathione Sepharose $4 \mathrm{~b}$ beads was added to the protein lysates and incubated for $1 \mathrm{~h}$ at $4{ }^{\circ} \mathrm{C}$. The beads were pelleted and washed for five times in cell lysis buffer. The eluted proteins were subjected to the immunoblotting.

\section{Immunohistochemistry and immunofluorescence assay}

For histology, tumor tissues were fixed with $4 \%$ paraformaldehyde at $4{ }^{\circ} \mathrm{C}$ overnight for paraffin embedding. After dewaxing, the slides were incubated in $3 \% \mathrm{H}_{2} \mathrm{O}_{2}$ for $20 \mathrm{~min}$ to block endogenous peroxidase. Antigen retrieval was done at $95{ }^{\circ} \mathrm{C}$ for $20 \mathrm{~min}$ with citric acid hydrochloric acid antigen retrieval buffer. Tissue sections were incubated with blocking buffer containing $5 \%$ BSA for 30 min and then subsequently with primary and secondary antibodies. SABC (BOSTER, Wuhan, China) was utilized to amplify signal before hematoxylin and AEC or DAB (ZSGB-BIO, Beijing, China) staining.

Cells grown on glass coverslips were analyzed with immunofluorescence assay as previous [13]. Briefly, the cells were fixed with $4 \%$ paraformaldehyde and permeabilized with $0.4 \%$ Triton X-100. After blocking with $3 \%$ BSA, the cells were incubated with primary antibodies and subsequently with fluorescein isothiocyanate (FITC)-labeled secondary antibodies. Coverslips were mounted with Prolong Gold Antifade Reagent (containing DAPI, Thermo, Hudson, USA). The signals were observed and photographed under the confocal microscopy (Nikon, ECLIPSE Ti).

\section{Tumor models}

Four-week-old male BALB/cA-nu mice were injected subcutaneously in the right underarms with Huh7 stable cells and Changliver stable cells $\left(5 \times 10^{6}\right.$ cells per $\left.100 \mu \mathrm{l}\right)$. After inoculation, tumor growth was monitored daily for 2-4 weeks with a vernier caliper until the tumor size reached approximately $100 \mathrm{~mm}^{3}$. Mice were sacrificed by spinal cord dislocation for tumor collection and subsequent detection at indicated time.

\section{TUNEL staining}

For TUNEL staining, mouse sh-TIPE2 tumor tissue and shCtrl/TIPE2 Huh7 cells were fixed with $4 \%$ paraformaldehyde, permeabilized with $0.1 \%$ Triton X-100, and stained with TUNEL labeling solution (Roche, Switzerland) according to the manufacturer's instructions. 


\section{Results}

\section{Downregulation of TIPE2 in tumor tissues of HCV-positive HCC}

To study the roles of TIPE2 in HCV-associated HCC, the expressions of TIPE2 in the HCV-positive HCC tissues were measured by immunoblotting. Ten HCVpositive $\mathrm{HCC}$ and their paracarcinoma tissues were applied to immunoblotting. As indicated in Fig. 1, the expression of TIPE2 is significantly reduced in eight of ten HCV-positive HCC tissues compared to that in the tissues adjacent to the HCC tissues (Fig. 1a). To exclude the contamination of other tissues or immune cells, immunohistochemistry was performed to confirm the downregulation of TIPE2 in hepatocyte (Fig. 1b). Similarly, lower level of TIPE2 was observed in the hepatoma cell lines such as Huh7 and HepG2 cells compared to non-cancer Changliver cells (data not shown). These results suggested that TIPE2 is involved in $\mathrm{HCV}$-associated HCC.

\section{HCV non-structural protein NS5A promotes TIPE2 degradation}

HCV promotes HCC development via encoding a number of oncoproteins such as Non-structural proteins NS3/4A, NS5A, and NS5B. To determine whether downregulation of TIPE2 in HCV-positive HCC tissue (Fig. 1) is regulated by HCV non-structural proteins, TIPE2 was transiently co-expressed with NS3/4A, NS5A, or NS5B in 293T cells. The immunoblotting results indicated that NS5A could significantly reduce TIPE2 protein expression level (Fig. 2a) in a dose-dependent manner (Fig. 2b). Furthermore, the downregulation of TIPE2 could be reversed by proteasome inhibitor, MG132 (Fig. 2c), which suggested that the degradation of TIPE2 induced by NS5A is proteasome dependent.

\section{NS5A interacts with TIPE2}

Since NS5A could reduce TIPE2 expression, we predict that there may be interactions between them. To test this
Fig. 1 Downregulation of TIPE2 in HCV-positive HCC tumor tissues. Representative photographs of TIPE2 protein expression in $\mathrm{HCV}$-positive $\mathrm{HCC}$ tissues $(\mathrm{Ca})$ and adjacent liver tissues $(\mathrm{N})$ examined by Western blot (a) and IHC (b) with antiTIPE2 antibody. Positive cells were stained in brown. Scale bars $=200 \mu \mathrm{m}$
A
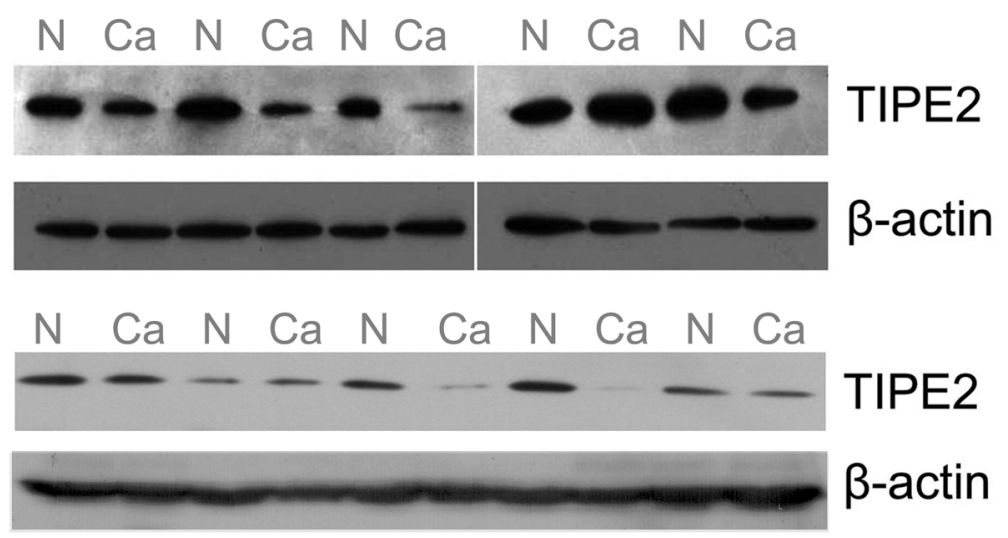

B

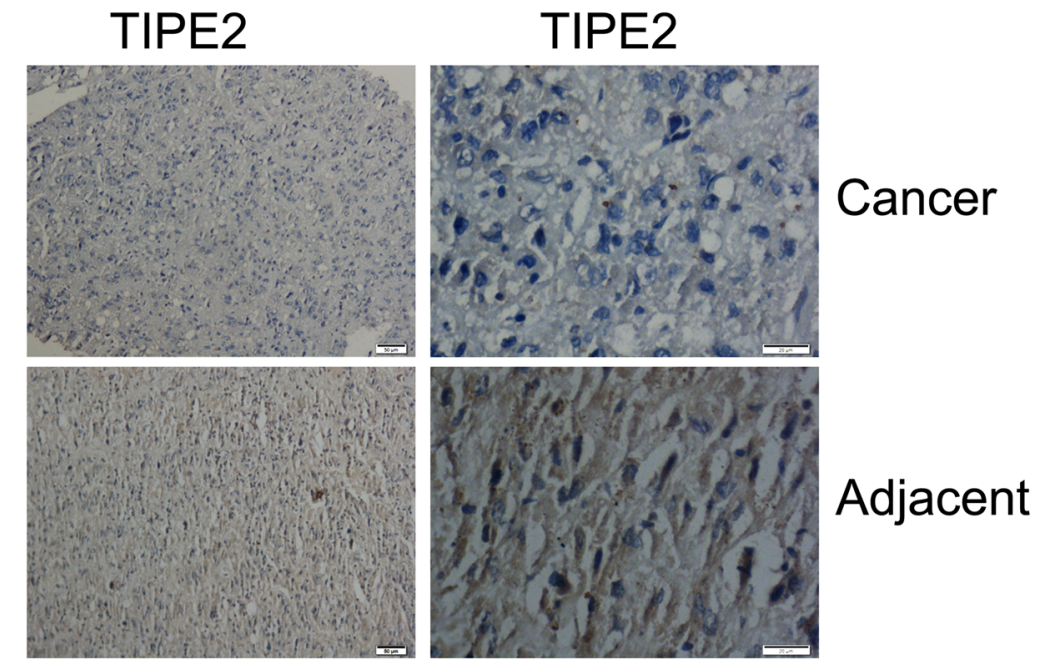


A

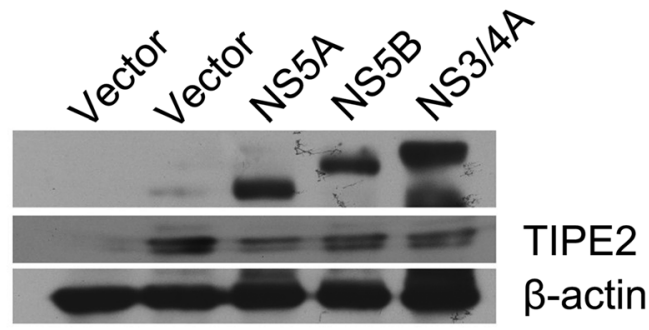

B

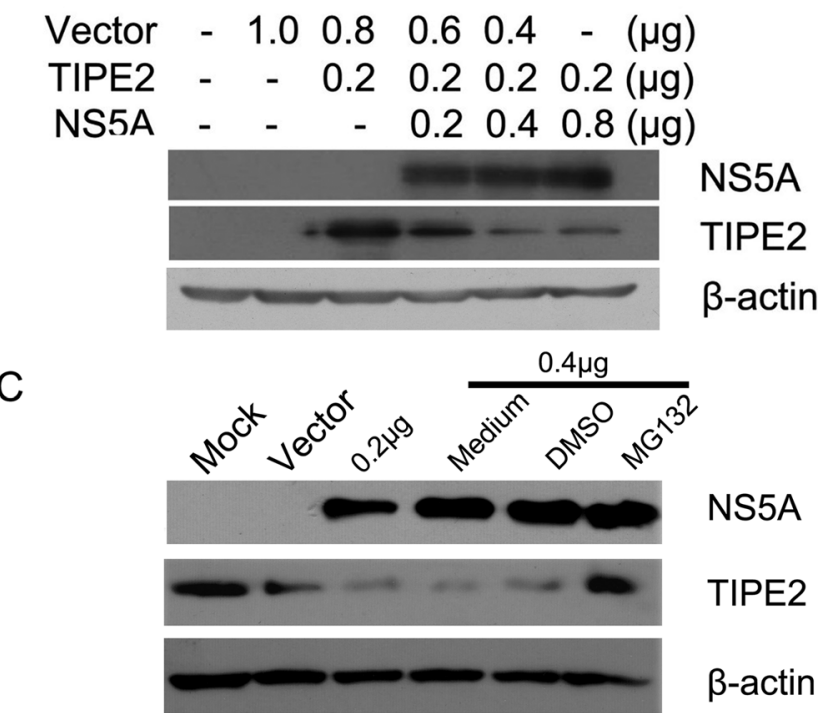

Fig. 2 HCV-encoded NS5A leads to TIPE2 degradation. a HEK293T cells were incubated in 12-well plate; when the confluence is up to $70 \%$, cells were transiently co-transfected with TIPE2 $(0.5 \mu \mathrm{g})$ and equal amount of HCV NS5A, NS5B, NS3/4A, or vector plasmids as indicated. Forty-eight hours post-transfection, expression of TIPE2 was determined by Western blot. b TIPE2 was co-transfected into $293 \mathrm{~T}$ cells with increasing amounts of NS5A plasmids. Forty-eight hours later, cell lysates were subjected to SDS-PAGE and analyzed by Western blot. c TIPE2 was co-transfected into 293T cells with increasing amounts of NS5A plasmids. Cells were treated with DMSO or $1 \mathrm{mM} \mathrm{MG132} 6 \mathrm{~h}$ before harvesting. Forty-eight hours post-transfection, cell lysates were subjected to SDS-PAGE and analyzed by Western blot

hypothesis, we constructed GST and GST-TIPE2 expression plasmids and expressed them in prokaryotic BL21 cells. The GST pull-down assay (Fig. 3a) revealed that TIPE2 was strongly associated with NS5A but hardly with NS5B or NS3/4A (Fig. 3a).

To further confirm the interaction between TIPE2 and NS5A, co-IP of myc-TIPE2 and FLAG-NS5A with antiFLAG antibody was performed. The result demonstrated a strong interaction between TIPE2 and NS5A (Fig. 3b). co-IP of FLAG-TIPE2 and myc-NS5A showed similar results (Fig. 3c). co-IP from Huh7 cells indicated that the interaction also exists in hepatoma cells (Fig. 3d).

To visualize the co-localization of TIPE2 and NS5A in situ, we co-transfected GFP-TIPE2 and myc-NS5A plasmids into $293 \mathrm{~T}$ cells. Confocal microscopy revealed an obvious co-localization of TIPE2 and NS5A in cytoplasm (Fig. 4a). The co-localization of TIPE2 and NS5A was also observed in Huh7 cells (Fig. 4b). Collectively, we concluded that NS5A binds TIPE2 and possibly leads to degradation of TIPE2.

\section{TIPE2 inhibits the DDR in vitro}

Cancer development is accompanied with continuous DNA damage. We therefore speculated that TIPE2 might be involved in DNA damage/repair. To determine whether TIPE2 is involved in tumor's DNA damage/repair, we generated Huh7-TIPE2-overexpressing stable cell line and Changliver-TIPE2 knockdown stable cell line by using lentivirus infection. The cells were left without treatment or treated with hydroxyurea (HU) for inducing DNA damage. The results indicated that ectopic expression of TIPE2 could reduce the phosphorylation level of H2AX, Chk1, and Chk2 in Huh7-TIPE2 cells (Fig. 5a). In contrast, the phosphorylation level of H2AX, Chk1, and Chk2 was much elevated in Changliver-TIPE2 knockdown cells (Fig. 5b). Consistently, we also observed the increase of TUNEL-positive cells in mice sh-TIPE2 tumor tissue and Huh7 stable cells (Fig. 5c). These results indicate that TIPE2 directly induces DNA damage and diminishes HU-induced DNA damage response.

\section{TIPE2 suppressed hepatocellular tumorigenesis in vitro and in vivo}

To examine the potential roles of TIPE2 in the inhibition of HCC tumor growth, we performed plate colony formation assay using Huh7-TIPE2 overexpression and Changliver-TIPE2 knockdown stable cell lines in vitro. As shown in Fig. 6a, almost no clone was formed by the Huh7-TIPE2-overexpressing cells. However, colony formation efficiency of Changliver-TIPE2 knockdown cells was significantly higher than that of the control cells. The results of Huh7-TIPE2 and Changliver-TIPE2 knockdown xenografts in nude mice suggest that the tumor volume and growth speed of Huh7-TIPE2 overexpression cells are much reduced compared to Huh7 itself (Fig. 6b-d). In contrast, the tumor growth and tumor volume derived from the Changliver-TIPE2 knockdown cells are much increased compared to the tumors of Changliver cells itself (Fig. 6b-d). Furthermore, we also observed the higher viability in TIPE2 stable Huh7 cells after NS5A overexpression (Fig. 6e). These results suggest that TIPE2 may negatively regulate HCC tumorigenesis.

\section{TIPE2 inhibits DDR in tumor}

To determine the molecular mechanism underlying the TIPE2 effect, we studied DNA damage response (DDR) in the tumors derived from the nude mice by immunohistochemistry. 
A
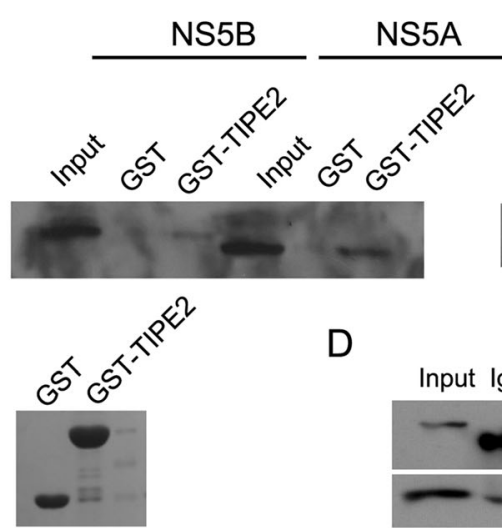

D
B

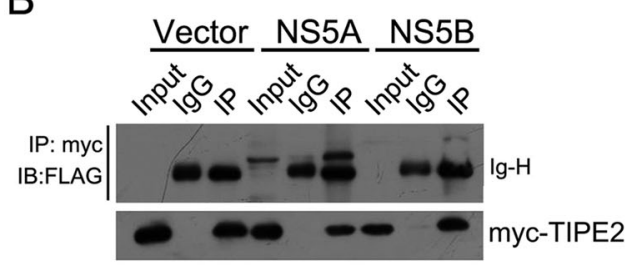

C

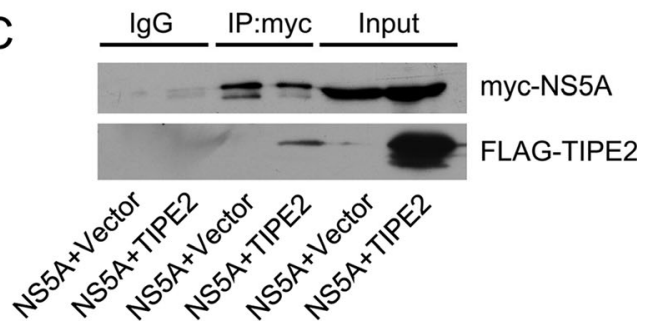

Fig. 3 Interaction between TIPE2 and NS5A. a The association of TIPE2 with NS5A, NS5B, or NS3/4A was detected by GST pull-down assay, and Coomassie Brilliant Blue staining of TIPE2 expressed in prokaryotic expression cells (BL21) was shown. b Myc-TIPE2 was transiently co-transfected into 293T cells with FLAG-NS5A or FLAGNS5B. Cell lysates were co-immunoprecipitated with anti-myc antibody

As shown in Fig. 7a, the expression of $\gamma-\mathrm{H} 2 \mathrm{AX}$ is much higher in the tumor tissue generated from Huh7 cells or isotype $\operatorname{IgG}$ followed by Western blot. c 293T cells were transiently transfected with FLAG-TIPE2 and myc-NS5A. The cell lysates were coimmunoprecipitated with anti-FLAG antibody or isotype IgG followed by Western blot. d Huh7 TIPE2 stable cells were seeded into 10-cm dish and transfected with $6 \mu \mathrm{g}$ myc-NS5A plasmids, and $48 \mathrm{~h}$ later, co-IP was performed as above
Fig. 4 Co-localization of TIPE2 and NS5A in cytoplasm. MycNS5As were co-transfected into 293T cells (a) or Huh7 cells (b) with EGFP or EGFP-TIPE2 plasmids, respectively. After $48 \mathrm{~h}$, the cells were fixed, stained, and analyzed with confocal microscopy
A

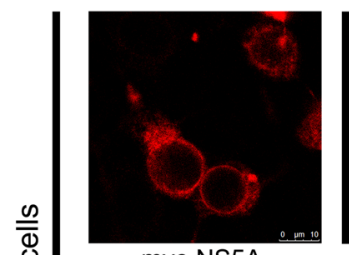

myc-NS5A

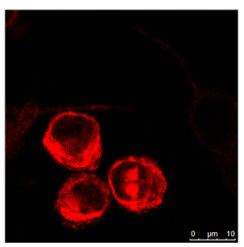

myc-NS5A

B

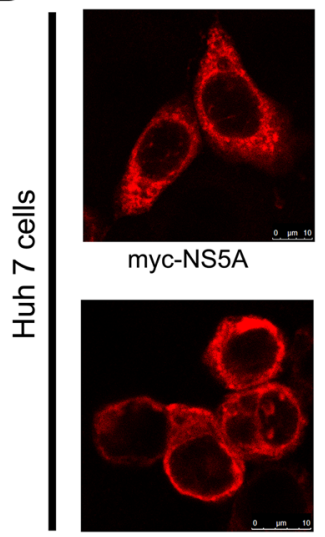

myc-NS5A

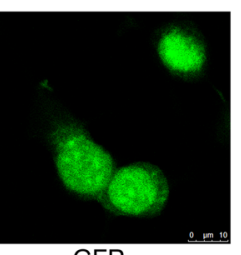

GFP

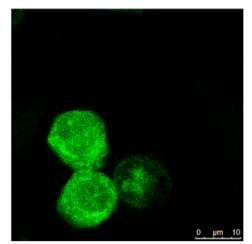

GFP-TIPE2

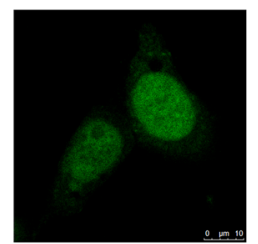

GFP

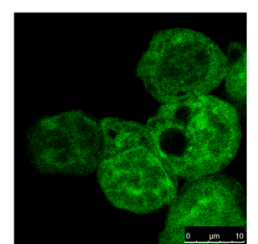

GFP-TIPE2

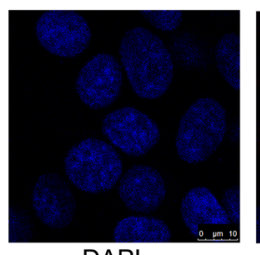

DAPI

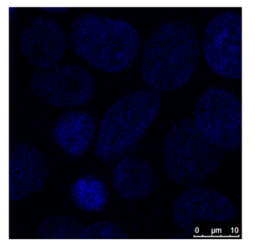

DAPI

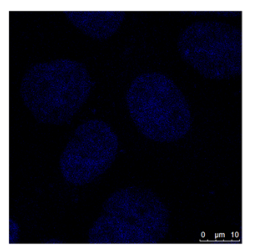

DAPI

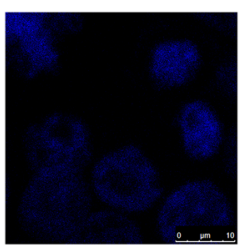

DAPI

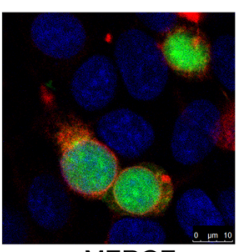

MERGE

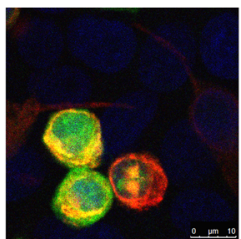

MERGE

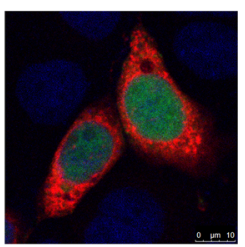

MERGE

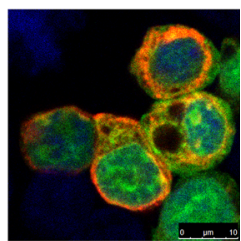

MERGE 
Fig. 5 Downregulation of TIPE2 stimulates DNA damage response. a TIPE2overexpressing stable cell line and control cell line were treated with hydroxyurea or DMSO as indicated. Cell lysates were collected and analyzed for phospho-H2AX, Chk1, and Chk2 levels with Western blot. b shTIPE2 and corresponding sh-Ctrl stable cell lines were exposed to hydroxyurea or DMSO, and then, the phospho-H2AX, Chk1, and Chk2 levels were determined by Western blot. c TUNEL staining was performed in mice sh-TIPE2 tumor tissue and Huh7 sh-TIPE2 stable cells. Green: TUNELpositive cells
A
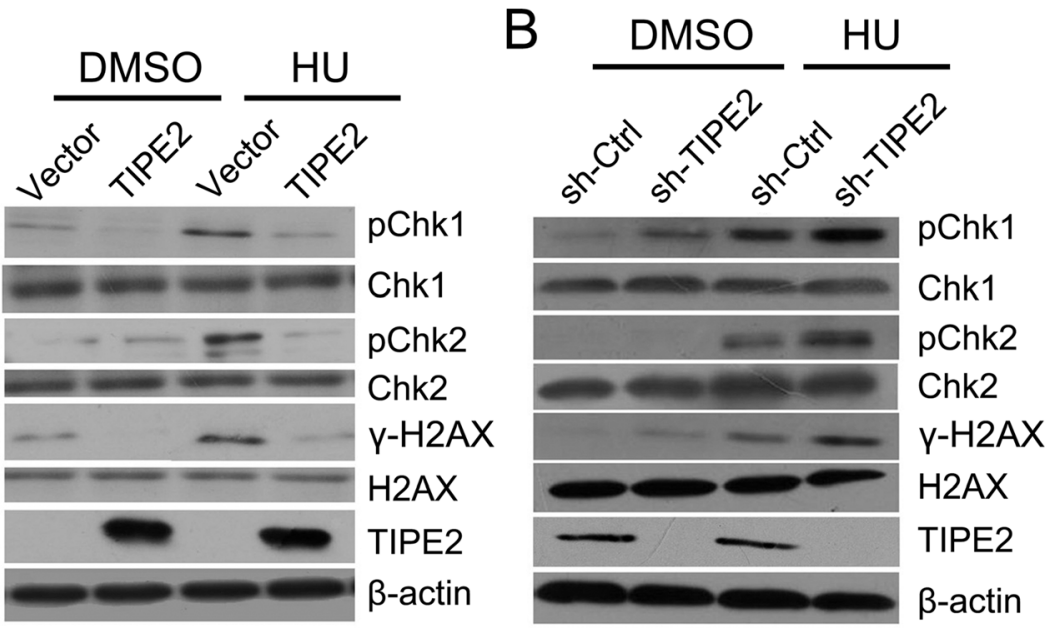

C
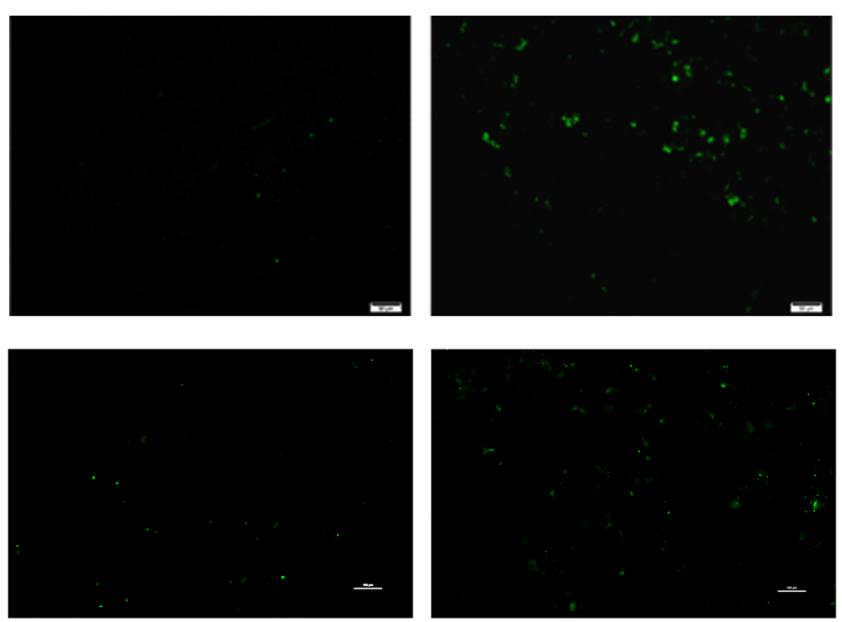

sh-Ctrl

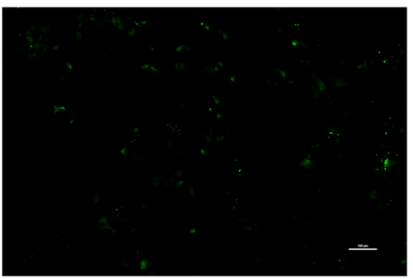

sh-TIPE2
$\mathrm{H} 2 \mathrm{AX}$ is much stronger in Changliver-TIPE2 knockdown tumors than that in the tumors generated from parental Changliver cell (Fig. 7b). These results strongly suggest that TIPE2 can inhibit tumorigenesis by suppressing the DNA damage response.

\section{Discussion}

TIPE2 is a newly identified regulator of immunity, while emerging evidence indicates that TIPE2 might be also a novel tumor suppressor. We therefore hypothesized that TIPE2 might be employed by cancer-causing pathogens, such as $\mathrm{HBV}$ and $\mathrm{HCV}$, to initiate tumorigenesis or promote tumor growth. Intriguingly, recent studies have reported that TIPE2 expression was decreased in the peripheral blood mononuclear cells (PBMCs) of patients with chronic hepatitis B and in HBV-related HCC tumor tissue [21, 23]. Compared to HBV, HCV infection is more strongly associated with cirrhosis, hepatic decompensation, and $\mathrm{HCC}$
[1]. In the present study, for the first time, we observed downregulation of TIPE2 in the HCV-positive $\mathrm{HCC}$ tissue from a cohort of patients. These data suggest that TIPE2 is a common pathway of virus-induced HCC development.

While downregulation of TIPE2 was noticed in HCC tissue, the mechanisms underlying it remain unknown. In the current study, we found that one of the HCV-encoded non-structural proteins NS5A was adversely associated with the expression of TIPE2. This suggests that NS5A leads to degradation of TIPE2. There are mainly two pathways leading to protein downregulation: transcriptional regulation and protein modification. Interestingly, no significant difference was observed at mRNA level of TIPE2 between HBV-related HCC and its adjacent non-tumor tissue, while obviously higher level of ubiquitination of TIPE2 was detected and the proteasome inhibitor MG132 could restore TIPE2 protein accumulation [18]. Those data suggest that TIPE2 was degraded at protein level in HCC. We also found that TIPE2 could be restored by MG132 at the presence of NS5A. The mechanism by 
Fig. 6 TIPE2 suppresses hepatocellular tumorigenesis in vitro and in vivo. a TIPE2overexpressing, TIPE2 silencing, and control cells were utilized for the plate colony formation assay, respectively. b Isolated tumors from mice injected with control, TIPE2-overexpressing, or TIPE2 silencing cells. c The average weights of isolated tumors in $\mathbf{b}$ were quantified. d The growth curves of subcutaneous tumors from the mice. e Cell viability was measured up to $48 \mathrm{~h}$ by MTT assay in TIPE2 stable expressing Huh7 cells after transient transfection with NS5A and control plasmids. $* p<0.05$
A

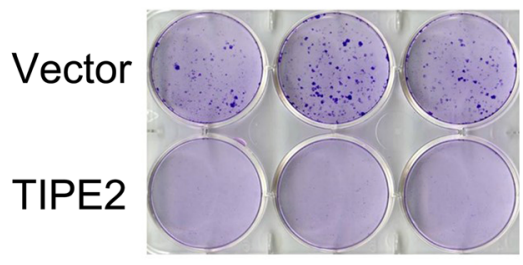

B
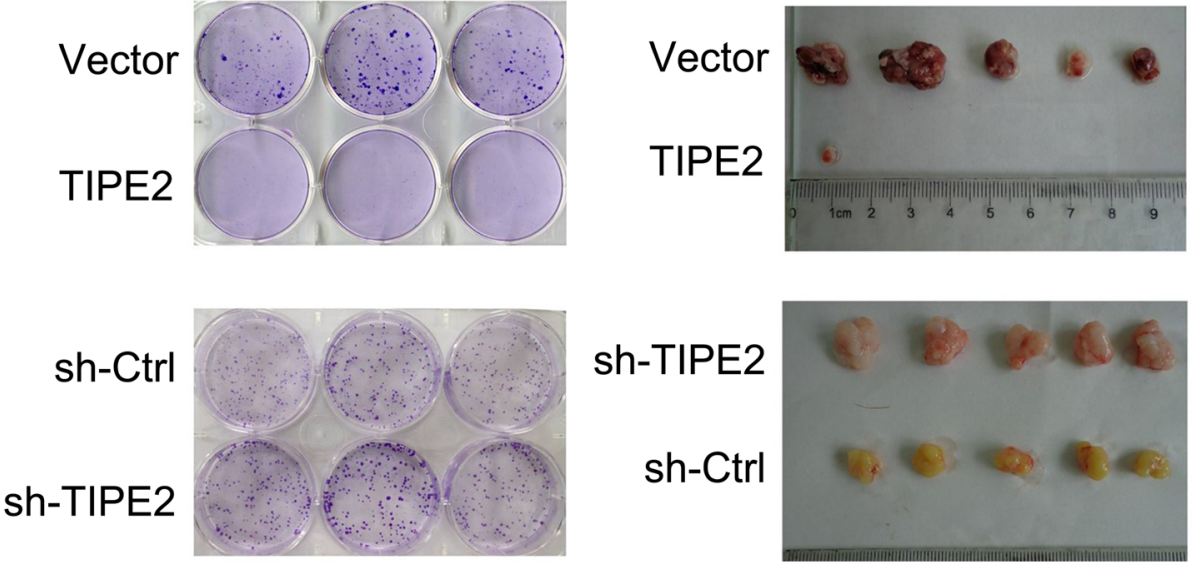

C
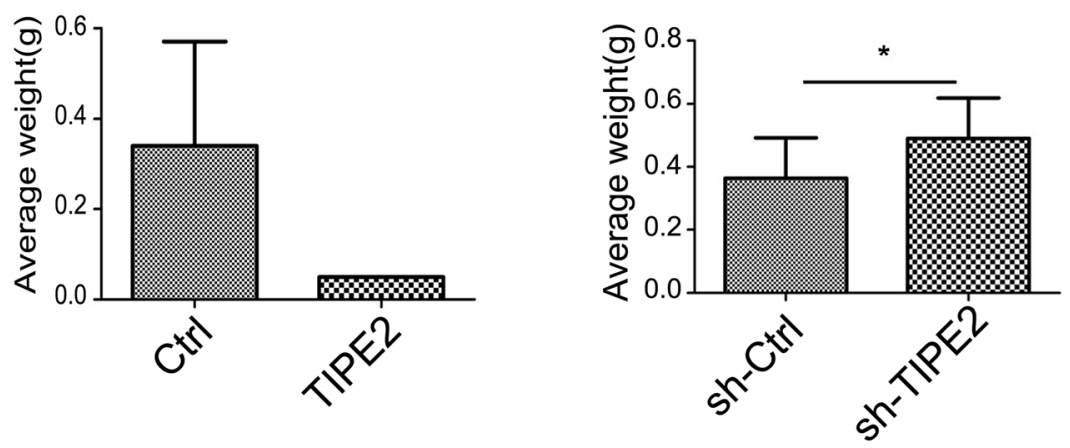

$\mathrm{D}$
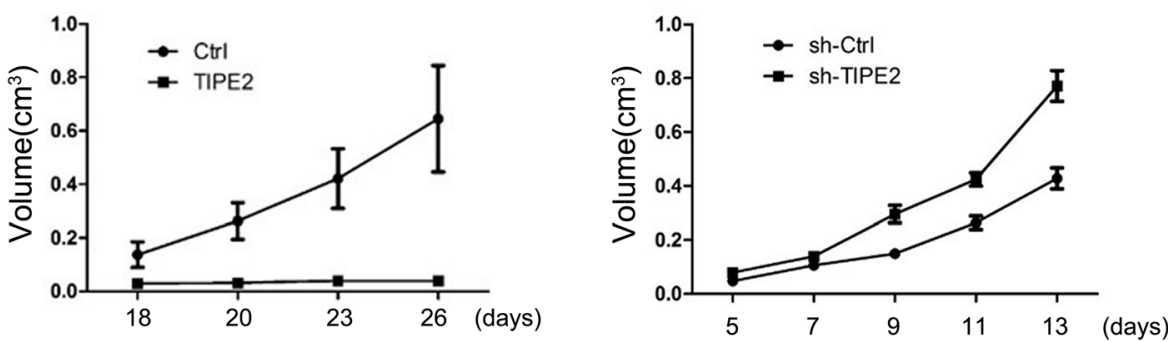

$E$

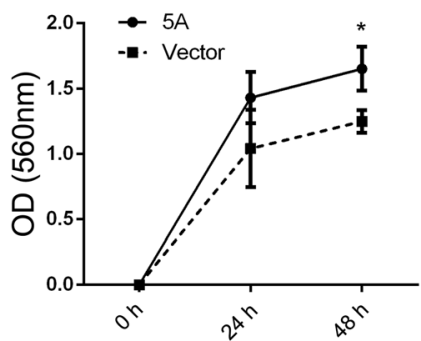

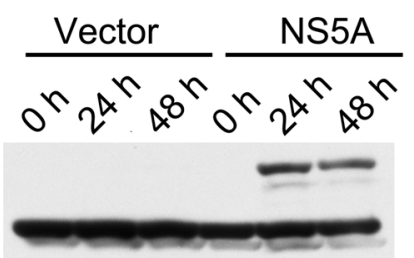

NS5A

$\beta$-actin which NS5A induces the degradation of TIPE2 is not clear and needs further investigation.

Reactive oxygen species (ROS) and the products of inducible nitric oxide synthase (NOS) generated upon HCV infection could lead to DNA damage [9, 24]. HCV-encoded core, $\mathrm{NS} 2, \mathrm{NS} 3 / 4 \mathrm{~A}$, and NS5B have been reported to trigger DNA damage. There have been also several studies that revealed association between NS5A and genomic instability via ROS.
High level of ROS could be induced by NS5A in transgenic mice, cooperated with upregulation of NF-KB and STAT3, which promote steatosis and HCC [25]. NS5A also reduced tumor suppressor PTEN expression in ROS-dependent pathway [26]. In fact, NS5A is associated with chromosomal instability and mitotic cell cycle dysregulation [10]. Interestingly, NS5A was recently shown to downregulate the growth arrest and DNA damage-inducible gene 45- $\alpha$ (GADD45 $\alpha$ ) 
Fig. 7 DNA damage in tumor tissues generated in the nude mice. Expression of $\gamma$-H2AX in the tumor tissues isolated from the mice injected with Huh7-TIPE2 and control cells (a) or Changliver sh-TIPE2 and control cells (b). Positive cells were stained in red in the nuclei. Scale bars $=50 \mu \mathrm{m}$
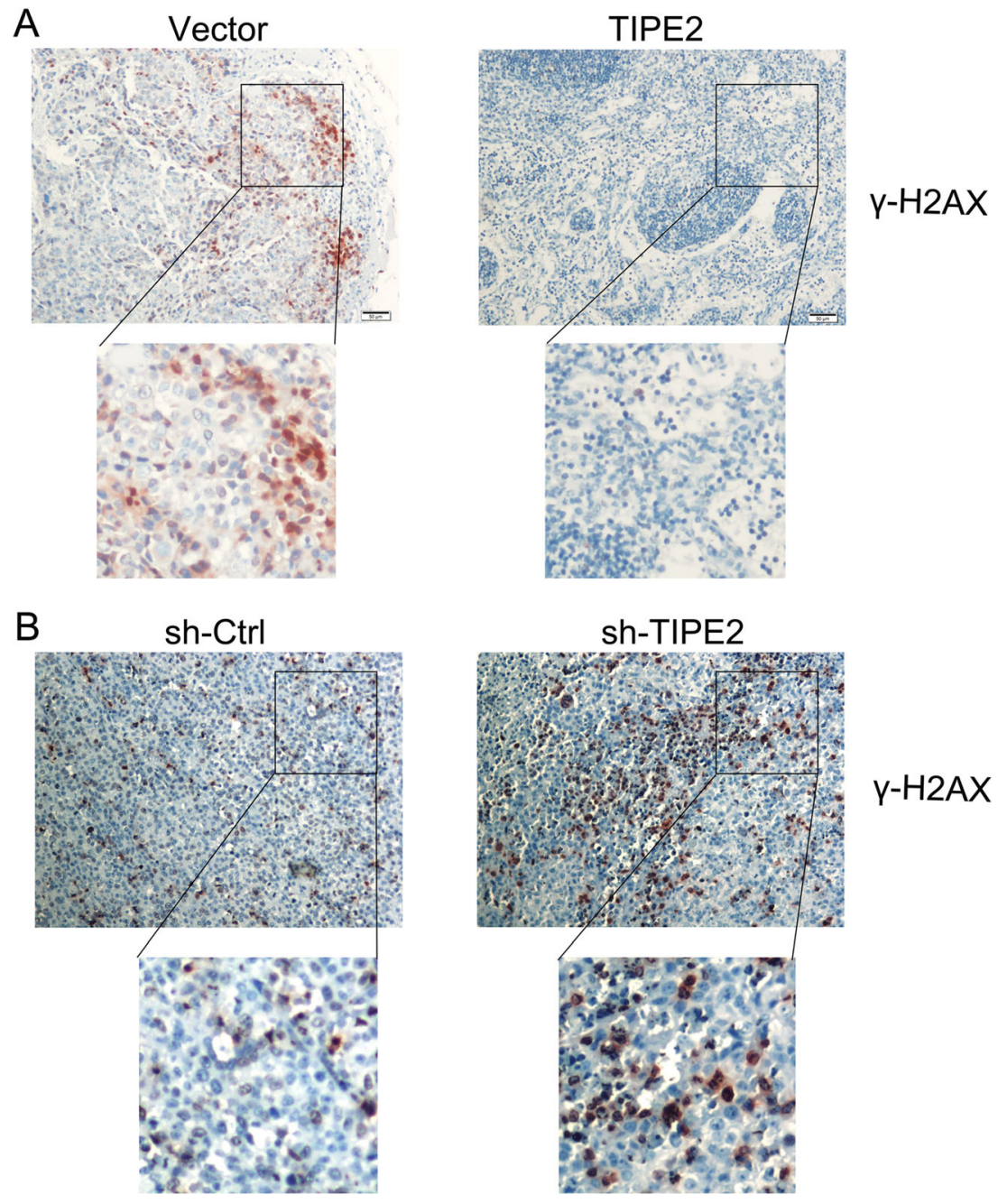

expression [27]. In this study, we showed that NS5A reduced TIPE2 expression and consequently induced DNA damage response. Activation of DNA damage response may result in impairment of damage repair, dysregulation of cell cycle, apoptosis, or tumor formation. Previous investigation has demonstrated that tumor is developed spontaneously in NS5A transgenic mice [28]. Our findings provide further evidence and molecular mechanism for the oncogenic effect of NS5A.

The connection between TIPE2 and DNA damage is surmisable according to the existing data. During infection, TIPE2 could inhibit phagocytosis and oxidative burst by binding to and blocking Rac GTPases [22]. Rac was reported to stimulate DDR. Furthermore, Rac1 was suggested responsible for the genotoxin-induced DNA damage in the liver [29]. On the other hand, Rac-mediated nuclear mechanisms are required for activation of DDR following topo II poison challenge in a p53- and heat shock protein-independent pathway [30]. So we predict that the upregulation of $\gamma$-H2AX, pChk1, and pChk2 in TIPE2 deficiency cells and $\mathrm{HCC}$ tumor tissue indicates that TIPE2 inhibits DDR during virus infection likely via the
Rac pathway. Taken together, we propose that TIPE2 is centralized in a pathogenic network of infection, inflammation, DNA damage, and tumor formation.

In summary, our work suggests that $\mathrm{HCV}$ promotes $\mathrm{HCC}$ development via DNA damage, through downregulation of TIPE2 by its encoded NS5A protein. Our study provides a novel mechanism underlying HCV-related HCC development and identifies TIPE2 as a potential therapeutic target for HCVrelated $\mathrm{HCC}$.

Acknowledgments The authors thank Dr. Wanli Ma for his help in collecting the tissue samples.

Compliance with ethical standards This study was approved by the Ethics Committees of the Medical College of Henan University, and written informed consent was obtained from all participants.

Conflicts of interest None

Funding This work was supported by NSFC grant 81402271 . 
Open Access This article is distributed under the terms of the Creative Commons Attribution 4.0 International License (http:// creativecommons.org/licenses/by/4.0/), which permits unrestricted use, distribution, and reproduction in any medium, provided you give appropriate credit to the original author(s) and the source, provide a link to the Creative Commons license, and indicate if changes were made.

\section{References}

1. Lin MV, King LY, Chung RT. Hepatitis C virus-associated cancer. Annu Rev Pathol. 2015;10:345-70.

2. Bosetti C, Turati F, La Vecchia C. Hepatocellular carcinoma epidemiology. Best Pract Res Clin Gastroenterol. 2014;28:753-70.

3. Fan JH, Wang JB, Jiang Y, Xiang W, Liang H, et al. Attributable causes of liver cancer mortality and incidence in China. Asian Pac J Cancer Prev. 2013;14:7251-6.

4. Ke PY, Chen SS. Hepatitis C virus and cellular stress response: implications to molecular pathogenesis of liver diseases. Viruses. 2012;4:2251-90.

5. Tanaka N, Moriya K, Kiyosawa K, Koike K, Gonzalez FJ, et al. PPARalpha activation is essential for HCV core protein-induced hepatic steatosis and hepatocellular carcinoma in mice. J Clin Invest. 2008;118:683-94.

6. Moriya K, Fujie H, Shintani Y, Yotsuyanagi H, Tsutsumi T, et al. The core protein of hepatitis $\mathrm{C}$ virus induces hepatocellular carcinoma in transgenic mice. Nat Med. 1998;4:1065-7.

7. Machida K, Tsukamoto H, Liu JC, Han YP, Govindarajan S, et al. c-Jun mediates hepatitis $\mathrm{C}$ virus hepatocarcinogenesis through signal transducer and activator of transcription 3 and nitric oxidedependent impairment of oxidative DNA repair. Hepatology. 2010;52:480-92.

8. Sakamuro D, Furukawa T, Takegami T. Hepatitis C virus nonstructural protein NS3 transforms NIH 3 T3 cells. J Virol. 1995;69:3893-6.

9. Machida K, Cheng KT, Lai CK, Jeng KS, Sung VM, et al. Hepatitis $\mathrm{C}$ virus triggers mitochondrial permeability transition with production of reactive oxygen species, leading to DNA damage and STAT3 activation. J Virol. 2006;80:7199-207.

10. Baek KH, Park HY, Kang CM, Kim SJ, Jeong SJ, et al. Overexpression of hepatitis C virus NS5A protein induces chromosome instability via mitotic cell cycle dysregulation. J Mol Biol. 2006;359:22-34.

11. Lai CK, Jeng KS, Machida K, Cheng YS, Lai MM. Hepatitis C virus NS3/4A protein interacts with ATM, impairs DNA repair and enhances sensitivity to ionizing radiation. Virology. 2008;370:295-309.

12. Machida K, McNamara G, Cheng KT, Huang J, Wang CH, et al. Hepatitis $\mathrm{C}$ virus inhibits DNA damage repair through reactive oxygen and nitrogen species and by interfering with the ATMNBS1/Mre11/Rad50 DNA repair pathway in monocytes and hepatocytes. J Immunol. 2010;185:6985-98.

13. Wang Y, Wang Y, Xu Y, Tong W, Pan T, et al. Hepatitis C virus NS5B protein delays $\mathrm{s}$ phase progression in human hepatocytederived cells by relocalizing cyclin-dependent kinase 2-interacting protein (CINP). J Biol Chem. 2011;286:26603-15.
14. Shawki SM, Meshaal SS, El Dash AS, Zayed NA, Hanna MO. Increased DNA damage in hepatitis $\mathrm{C}$ virus-related hepatocellular carcinoma. DNA Cell Biol. 2014;33:884-90.

15. Hoare M, Shankar A, Shah M, Rushbrook S, Gelson W, et al. Gamma-H2AX+CD8+ T lymphocytes cannot respond to IFN-alpha, IL-2 or IL-6 in chronic hepatitis C virus infection. J Hepatol. 2013;58:868-74.

16. Sun H, Gong S, Carmody RJ, Hilliard A, Li L, et al. TIPE2, a negative regulator of innate and adaptive immunity that maintains immune homeostasis. Cell. 2008;133:415-26.

17. Zhang X, Wang J, Fan C, Li H, Sun H, et al. Crystal structure of TIPE2 provides insights into immune homeostasis. Nat Struct Mol Biol. 2009;16:89-90.

18. Gus-Brautbar Y, Johnson D, Zhang L, Sun H, Wang P, et al. The anti-inflammatory TIPE2 is an inhibitor of the oncogenic Ras. Mol Cell. 2012;45:610-8.

19. Li Y, Li X, Liu G, Sun R, Wang L, et al. Downregulated TIPE2 is associated with poor prognosis and promotes cell proliferation in non-small cell lung cancer. Biochem Biophys Res Commun. 2015;30:43-9.

20. Zhang YH, Yan HQ, Wang F, Wang YY, Jiang YN, et al. TIPE2 inhibits TNF-alpha-induced hepatocellular carcinoma cell metastasis via Erk1/2 downregulation and NF-kappaB activation. Int $\mathrm{J}$ Oncol. 2015;46:254-64.

21. Xi W, Hu Y, Liu Y, Zhang J, Wang L, et al. Roles of TIPE2 in hepatitis $\mathrm{B}$ virus-induced hepatic inflammation in humans and mice. Mol Immunol. 2011;48:1203-8.

22. Wang Z, Fayngerts S, Wang P, Sun H, Johnson DS, et al. TIPE2 protein serves as a negative regulator of phagocytosis and oxidative burst during infection. Proc Natl Acad Sci U S A. 2012;109:154138.

23. Zhang W, Zhang J, Zhao L, Shao J, Cui J, et al. TIPE2 protein negatively regulates $\mathrm{HBV}$-specific $\mathrm{CD} 8(+) \mathrm{T}$ lymphocyte functions in humans. Mol Immunol. 2015;64:204-9.

24. Higgs MR, Chouteau P, Lerat H. 'Liver let die': oxidative DNA damage and hepatotropic viruses. J Gen Virol. 2014;95:991-1004.

25. Wang AG, Lee DS, Moon HB, Kim JM, Cho KH, et al. Nonstructural $5 \mathrm{~A}$ protein of hepatitis $\mathrm{C}$ virus induces a range of liver pathology in transgenic mice. J Pathol. 2009;219:253-62.

26. Cheng D, Zhang L, Yang G, Zhao L, Peng F, et al. Hepatitis C virus NS5A drives a PTEN-PI3K/Akt feedback loop to support cell survival. Liver Int. 2015;35:1682-91.

27. Cheng D, Zhao L, Zhang L, Jiang Y, Tian Y, et al. p53 controls hepatitis $\mathrm{C}$ virus non-structural protein $5 \mathrm{~A}$-mediated downregulation of GADD45alpha expression via the NF-kappaB and PI3KAkt pathways. J Gen Virol. 2013;94:326-35.

28. Gimenez-Barcons M, Wang C, Chen M, Sanchez-Tapias JM, Saiz $\mathrm{JC}$, et al. The oncogenic potential of hepatitis C virus NS5A sequence variants is associated with PKR regulation. J Interf Cytokine Res. 2005;25:152-64.

29. Bopp A, Wartlick F, Henninger C, Kaina B, Fritz G. Rac1 modulates acute and subacute genotoxin-induced hepatic stress responses, fibrosis and liver aging. Cell Death Dis. 2013;4:e558.

30. Wartlick F, Bopp A, Henninger C, Fritz G. DNA damage response (DDR) induced by topoisomerase II poisons requires nuclear function of the small GTPase Rac. Biochim Biophys Acta. 2013;1833: 3093-103. 